\title{
The Crystal Field Plasmon Splitting
}

\author{
Stefano Antonio Mezzasalma, ${ }^{\dagger+}$ Marek Grzelczak, ${ }^{\circ *}$ Jordi Sancho-Parramon $^{\dagger *}$ \\ † Materials Physics Division, Ruđer Bošković Institute, Bijenička cesta 54, 10000 Zagreb, Croatia \\ * Lund Institute for advanced Neutron and X-ray Science (LINXS), Lund University, \\ IDEON Building: Delta 5, Scheelevägen 19, 22370 Lund, Sweden; \\ - Centro de Física de Materiales CSIC-UPV/EHU and Donostia International Physics Center (DIPC), \\ Manuel Lardizabal Ibilbidea 5, 20018 Donostia-San Sebastián, Spain
}

\begin{abstract}
We present an electromagnetic analogue of crystal (or ligand) field theory that describes geometric eigenmodes and the resonant plasmon wavelength in plasmonic nanocrystals in terms of a simple shape descriptor. Our model - crystal field plasmon splitting - is based on secular equations for geometric eigenmodes, allowing for the separation of pure shape from materials effects. As an example, the model is implemented to the experimental gold nanoparticles that undergo cube-to-sphere transition, showing that geometric eigenvalues change correspondingly in analogy to the atomic energy levels in Tanabe-Sugano correlation diagrams.
\end{abstract}

\section{Keywords: Plasmonics, Crystal Field Theory, Gold Nanoparticles, non-Retarded and Retarded Theory, Excess Charge Correction.}

Plasmonics arises from a complex interplay of electronic and geometric properties, where size and shape ${ }^{1}$ chemical composition, ${ }^{2}$ surface charge ${ }^{3}$ and dielectric environment ${ }^{4}$ turn out to dramatically affect localized surface plasmon resonance (LSP) and absorption efficiency in plasmon-induced phenomena such as hot carriers injection, ${ }^{5,6}$ radiative and resonance energy transfers. ${ }^{7}$ While the relevance of topology was well established in electronic structure theory, ${ }^{8}$ a little is known about purely topological shape effects in nanoplasmonics. The notion of shape, standing in between geometry and topology, is used therein mostly in connection with the (differential) geometry of surfaces, e.g. SP in curved media, scattering and radiation at bends and interfaces. ${ }^{9-12}$ Topology optimization of nanostructures ${ }^{13}$ can be regarded as well as an unrestricted shape optimization of a geometric functional.

A primary shape effect in LSP resonance may be detected by the so-called figure of merit, the ratio between enhanced local and incident fields, whose formal dependence on the real and imaginary parts of the complex dielectric function is changing with the particle shape. ${ }^{14}$ The linear optical response then may be derived from Drude's model or some semiclassical extension of it, ${ }^{15,16}$ aiming to include the relevant electron relaxation and transition processes implied by the metal band structure. Such features, clearly, are still material- and chemistry-mediated, with the problem that electronic Schrödinger's equations for particles of arbitrary shape may demand explicit solutions for very large quantum numbers. On the other hand, since the time of De Witt and da Costa, ${ }^{17,18}$ surface geometry is known to affect the motion both of classical waves and quantum objects. A number of studies ${ }^{19-21}$ were foreseeing the existence of curvature-driven topology eigenstates, inducing quantum interference phenomena and influencing surface charge transmission in/out a nanostructure. As an example, when optical waves squeeze onto a curved thin dielectric layer (a film waveguide), a frictional energy of geometric nature was deduced in the wave-optics domain to act either as a potential well or barrier, according to the sign of surface concavity. ${ }^{22,23}$ On this basis, it seems to be plausible that damping sources of topological origin may partake in the energy transfer elicited by nanoparticles (plasmonic hot carriers, photocatalytic processes, etc.). Shape effects on individual plasmonic particles are also essential to understand the interaction of plasmon resonances in complex structures. In this context, a sphere to cube transition may significantly quench the excitation of Fano resonances in coupled nanoplate-nanoshere systems. ${ }^{24}$

In this work, we link the topological shape effects in plasmonic nanoparticles with crystal field theory (CFT) that rests on topology and group theory. To separate pure shape from materials effects, we implemented the framework of secular equations for geometric eigenmodes. ${ }^{25,26}$ They define the complete basis set of naturally self-sustained multipoles with no external sources, in which the inhomogeneous term of the full electrostatic equation can be represented. The theoretical section thus devises a formalism which then is applied to sphere-to-cube plasmonic transition in experimental gold nanoparticles. The CFT model is employed either in a retarded or non-retarded (quasistatic) picture.

Cube-to-sphere transition in gold nanoparticles. Because this work deals with a novel theoretical framework for plasmonic shape effects, it is of the utmost importance resorting to a synthesis scheme which is able of tuning shape variations with high accuracy. We selected cube-like Au nanoparticles (40, 50, and 60 nm sized, Figure 1a - upper row) that were subjected towards oxidative etching. ${ }^{27}$ The oxidation occurs at metal surface sites with larger curvature (edges), leading to a gradual rounding to eventually produce nearly spherical nanoparticles (Figure 1a - bottom row). The initial and partially oxidized nanocubes as well as fully rounded nanoparticles are referred hereinafter as cubes, semicubes and spheres, respectively (Figure 1a). The analysis by Transmission Electron Microscopy confirmed that the mean particle length $\langle L\rangle$ decreases upon the oxidation process (Figure 1c). Interestingly, the extent of size reduction upon etching is more pronounced for larger particles, which is due to the well-defined cube-like morphology (higher shape anisotropy) of larger cubes, as compared to the smaller ones, displaying more rounded edges. To obtain the values of length $(L)$ and circularity $(C)$ for each sample, an analysis of TEM images was conducted by using Fiji 
software. As $\mathrm{C}$ is proportional to the ratio between particle area and squared perimeter, some geometric analysis allowed us to connect it to the shape descriptor used throughout this study, defined in terms of particle length and radii of spherical caps $(R)$ as $\mathrm{S}=1-\frac{2 R}{L}$ (Figure 1d). A further statistical propagation error was performed to correct the obtained data in light of the uncertainty related to the analysis of TEM images and to get the best predictions for S. Finally, the mean and standard deviation of the particle radius follow under the assumption of a theorem on the aleatory product distribution for two Gaussian variables (i.e. $L$ and $R / L$ ). All collected values are gathered in Table S1 of SI section 2 .

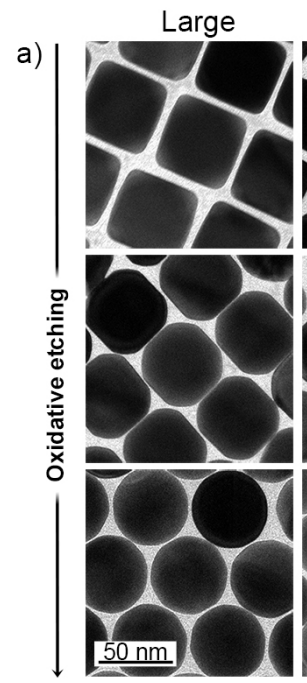

b)

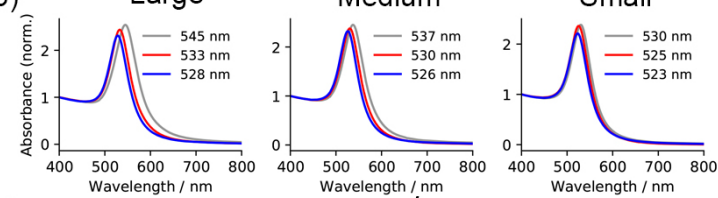

c)

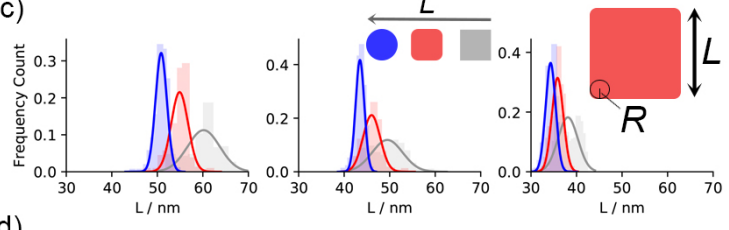

d)
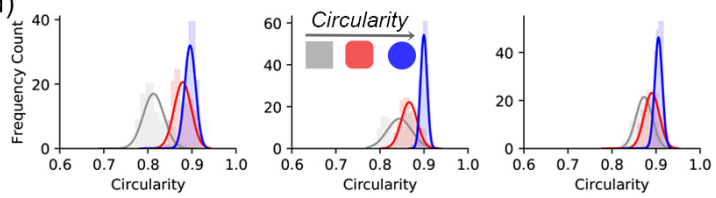

Fig. 1 Shape transition from cubes to spheres for large, medium and small Au nanoparticles. a) TEM images of initial Au nanocubes (top row), semicubes obtained after mild oxidation (middle row), and spheres after moderate oxidation (bottom row). All images share the same scalebar. b) UV-VisNIR spectra of all nanoparticles showing gradual LSP blueshift upon oxidative etching. c) All length distributions versus oxidative etching. d) Circularity change for large, medium and small nanoparticles showing that circularity values increase upon the cube-to-sphere transition.

Geometric normal modes. In a quasistatic picture, neglecting retardation but preserving a frequency-dependent dielectric function $(\epsilon)$, surface charges follow from the applied and induced ex- ternal fields. ${ }^{26,28}$ Normal modes are characterized by a family of eigenvalues $\left(\lambda_{\underline{k}}\right)$ and eigenfunctions $\left(\sigma_{\underline{k}}\right)$ that only depend on the geometric shape and can be represented by a multipole expansion with Fourier's coefficients $\underline{k}=\{\ell, m\}$. Figure (2) shows their evolution $(\ell \leq 5)$ when a sphere is transformed into a cube by varying the shape descriptor S. Between the extremes, (sphere) $0<\mathrm{S}<1$ (cube), lies a continuous class of semicubes with cylindrical contours, uniquely defined by the value of $\mathrm{S}$.

We performed numerical simulations by using the MNPBEM code $^{29}$ that relies on the boundary element method (SI section 1 - Numerical), ${ }^{26,30}$ returning a discrete set of $\sum(2 \ell+1)=35$ normal modes, corresponding to the most stable states. For a sphere, they are given by spherical multipoles, each with degeneracy $2 \ell+1$ ( $m=-\ell \ldots \ell$, see next equation 2$)$. Their individual evolution for $S \neq 0$ shows a degeneracy breaking and defines in the end a sort of band structure. However, tracking the mode evolution at a fixed $\ell$ is not supplied in MNPBEN by a direct toolbox output. As eigenvalues are ordered by their value, and not by the multipole degree, it was necessary to introduce some analytical criterion to discriminate among them. We found that imposing the numerical continuity to eigenvalues and their first derivative with respect to $\mathrm{S}$ was very efficient both to sort out different bands and to follow individual modes. Results were clearly reversible, i.e. fully reproducible upon reverting the transition, i.e. $S=0 \rightarrow 1$ or $\mathrm{S}=1 \rightarrow 0$.

Note that dipole $(\ell=1)$ is the only one always retaining the same degeneracy, while the quadrupole of the sphere $(\ell=2)$ splits into a three-fold corner mode and a two-fold edge mode (Figure 2). Octupole $(\ell=3)$, on the other hand, is sent into three bands, a non-degenerate corner mode, a three-fold edge mode, and a three-fold corner-edge mode. Sphere eigenvalues monotonically increase with increasing multipole degree, whereas the three most stable cube modes are corner-like, ${ }^{31}$ displaying dipolar, quadrupolar and octupolar nature (Figure 2 and SI section $3)$.

Predicting the optical spectra. To calculate the extinction cross sections we used the MNPBEM toolbox under quasistatic and fully retarded approximation schemes and using particle size and shape descriptor that came from the statistical analysis in SI sections $(2 \mathrm{~A}, 2 \mathrm{~B}, 2 \mathrm{C})$. Optical response, which is isotropic as a consequence of geometric symmetry, turns out to be mostly dominated by dipole-like corner modes. Note that for Ag nanoparticles larger higher-order contributions to LSP spectra are visible (SI section 4).

Simulated spectra were systematically red-shifted by a number of nanometers in comparison with experimental spectra. In general, the larger and less spherical the particles were, the larger the shift magnitude was. Both the electrolyte-induced reduction of the solution dielectric function and polydispersity effects were ruled out as possible causes for this anomalous red-shift (SI sections 5D,5E). A suitable explanation can be given instead by some excess charge at the $\mathrm{Au}$ /solution interface, implying a larger effective plasma frequency for Au particles that blue-shifts the plasmon resonance. Surface charge mechanisms result from a complex interplay of intermolecular forces and can be well quantified by thorough thermodynamic models. ${ }^{32}$ The shift of the ratio 


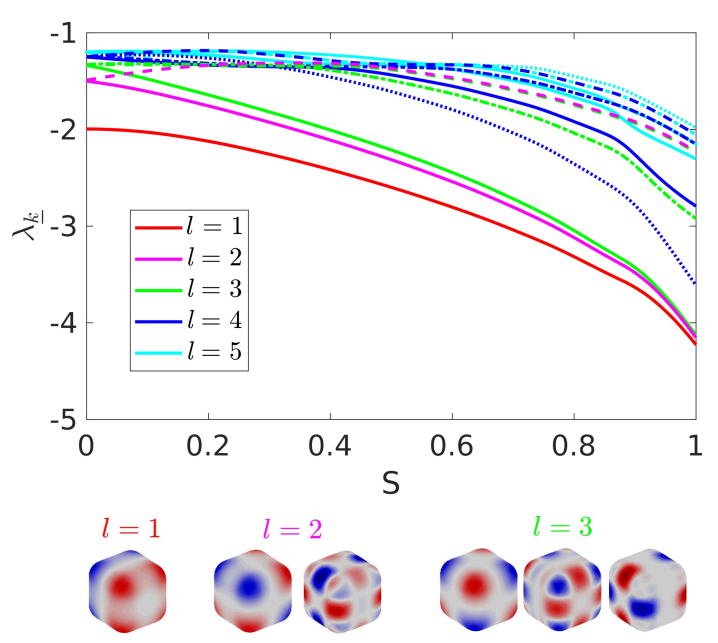

Fig. 2 Eigenvalues $(1 \leq \ell \leq 5)$ as a function of shape descriptor $(\mathrm{S}=0,1$ for perfect spheres and cubes, respectively). Different bands with equal $l$ are plotted using different line types and having the same colour. A space representation of modes for up to $l=3$ is also reported in the lower figure panel for the semicube with $\mathrm{S}=\frac{1}{2}$, their degeneracy conforming to equations (6-8).

between effective and bulk plasma frequencies may be either described by a linear dependence in the particle specific surface or in the shape descriptor (SI section 5F). By this linear law, and a standard procedure endowed with electronic interband transitions, ${ }^{33}$ the Au dielectric function was recalculated with corrected plasma frequencies. The final comparison among experiments and computations is depicted in Table (1), proving a satisfactory agreement. A synopsis of all experimental and numerical results is reported instead in Table (S2) of SI section (5G), where it may be seen that quasistatic predictions exceed the experimental values and turn out to be very close to corrected-retarded data (except for $\mathrm{S}=0.81$ ). While this is confirming the need to correct retardation here, a quantitative explanation of this occurrence is not yet ready and is left for future work. Qualitatively, an available excess charge may tend to further balance the field across the particle.

Table 1 Summary of LSP resonance predictions. Theoretical (th) and experimental (ex) wavelengths $t$ are in $\mathrm{nm}$.

\begin{tabular}{lll|lll|lll}
\hline $\mathrm{S}$ & $\lambda(\mathrm{ex})$ & $\lambda(\mathrm{th})$ & $\mathrm{S}$ & $\lambda(\mathrm{ex})$ & $\lambda(\mathrm{th})$ & $\mathrm{S}$ & $\lambda(\mathrm{ex})$ & $\lambda(\mathrm{th})$ \\
\hline 0.51 & 530 & 531 & 0.67 & 537 & 540 & 0.81 & 545 & 557 \\
0.41 & 525 & 528 & 0.58 & 530 & 534 & 0.55 & 533 & 534 \\
0.26 & 523 & 524 & 0.38 & 526 & 524 & 0.44 & 528 & 527 \\
\hline
\end{tabular}

Figure 3 illustrates the uncorrected and corrected spectra (without and with excess charge) versus measurements. The excellent agreement for the simulated widths confirms as well the accuracy of the low-loss imaginary part of the adopted dielectric function (Au single crystals). ${ }^{34}$ If other widely used optical constants were assumed, ${ }^{35}$ the predicted width would be larger than the experimental one. To prove this conclusion, SI section (5F) compares the results from single crystal and Johnson \& Christy's optical data for Au.

Secular equation and sphere eigenvalues from vanishing electrostatic energy. The study of electrostatic normal modes, for a metal particle of arbitrary shape $(p)$ in a dielectric medium $(d)$, is basically a boundary condition problem, where eigenvalues are expressible by the ratio of the frequency-dependent dielectric constants, $\lambda \equiv \epsilon_{p}(\omega) / \epsilon_{d}(\omega)$. The condition $\lambda_{\underline{k}}<0$ translates the selfsustainability of natural oscillations, the energy maintaining the field externally being balanced by an equal amount of internal work, or vice versa. ${ }^{36}$ This limiting constraint identifies topology spectra that are only a signature of shape as, in the longwavelength limit, retardation is negligible and transverse modes do not contribute to absorption.

Formally, surface modes take place when the polarization field inside the particle obeys the joint longitudinal and transverse condition, $\nabla \cdot \mathbf{P}=\nabla \wedge \mathbf{P}=0$, while a polarization charge $\nabla \cdot \mathbf{P} \neq 0$ develops at the surface. In the language of potential theory, equivalent single-layer or double-layer representations may be afforded respectively for the (internal) electric potential ${ }^{37}\left(V_{i}\right)$ or the surface charge density $(\sigma):^{38}$

$$
\int \sigma_{\underline{k}}\left(\mathbf{r}^{\prime}\right) \partial_{\mathbf{n}} \frac{1}{\left|\mathbf{r}-\mathbf{r}^{\prime}\right|} d s^{\prime}=\Lambda_{\underline{k}} \sigma_{\underline{k}}(\mathbf{r})
$$

$\partial_{\mathbf{n}}$ being the normal derivative at the surface and $\left|\mathbf{r}-\mathbf{r}^{\prime}\right|^{-1}$ denoting the static limit of Green's function with observation point $\mathbf{r}$. The (real-valued) eigenvalues, $\Lambda_{\underline{k}}=2 \pi\left(1+\lambda_{\underline{k}}\right) /\left(1-\lambda_{\underline{k}}\right)$, can be linked to the secular equation in frequency domain provided an optical dispersion law is stated for the dielectric function. When Mie's theory is applied to a sphere, for a given plasma frequency $\left(\omega_{\mathrm{p}}\right)$ and angular momentum $(\ell)$ of a plasmon mode, one has $\omega_{\ell}^{2} / \omega_{\mathrm{p}}^{2}=$ $\ell /(2 \ell+1) .{ }^{39}$ Drude's model then allows to recover:

$$
\lambda_{\underline{k} s}=-1-1 / \ell \quad(\text { sphere })
$$

We have proven in SI section (6H) that the eigenvalues of spherical harmonics $Y_{\ell m}(\theta, \varphi)(\theta, \varphi=$ polar, azimuth angles) in equation (2) also stem from the flux equation for the fields internal $(i)$ and external $(e)$ to the particle at a vanishing electrostatic energy $(E=0):^{36}$

$$
8 \pi E=\epsilon_{p} \int V_{i}(\mathbf{r}) \nabla V_{i} \cdot d \mathbf{s}-\epsilon_{d} \int V_{e}(\mathbf{r}) \nabla V_{e} \cdot d \mathbf{s}
$$

i.e.:

$$
\lambda=\frac{\int V_{e} \partial_{\mathbf{n}} V_{e} d s}{\int V_{i} \partial_{\mathbf{n}} V_{i} d s}
$$

which was generalized in terms of two Green functions $\left(\mathrm{G}_{i, e}\right)$, defined at both surface sides for each $\underline{k}$ (see equation $\mathrm{H} 2$ of SI section $6 \mathrm{H}$ ). Note that, for negative eigenvalues to be attained, the signs of normal gradients should be opposite, no matter which specific convention may be adopted. The next applications of equation (4) thus will stick for simplicity to equations (H4) of SI section $(6 \mathrm{H})\left(\partial_{\mathrm{n}} \mathrm{G}_{i}>0\right.$ and $\left.\partial_{\mathrm{n}} \mathrm{G}_{e}<0\right)$.

Plasmon resonance in semicubes from crystal field splitting. Deforming a sphere into a highly spherical semicube $\left(\mathrm{S} \rightarrow 0^{+}\right)$reduces the particle symmetry, leading eigenvalues to a degeneracy removal. ${ }^{40}$ The phenomenology subtended by Figure (2) will be explained by CFT, pioneered long ago by Bethe ${ }^{41}$ to model coordination complexes. Evolution of edges, faces and corners will be described by a minimal model of effective surface charges, mimicking the topology transition by a suitable charge redistribution. 


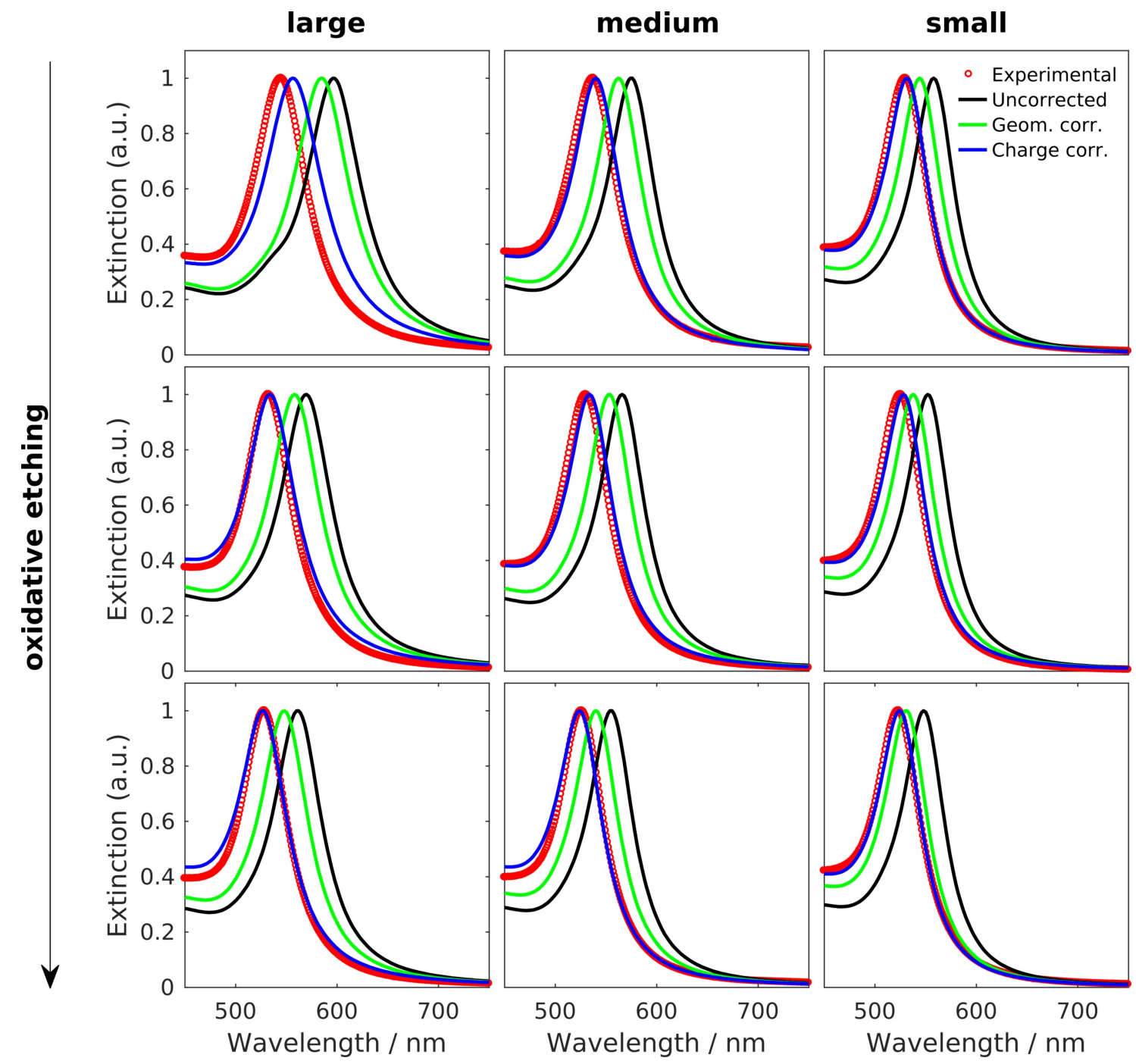

Fig. 3 Experimental and simulated optical extinction spectra for all samples, corresponding to the TEM images in Figure (1). Simulations with uncorrected (black), geometrically corrected (green) and charge corrected (blue) are superimposed to the experimental data (red) to show the proper trend of the approximations made and the accuracy of the final prediction.

To fix the ideas, let a $\mathrm{S}=0 \rightarrow 0^{+}$. At a given $\underline{k}=(\ell, m)$, each eigenvalue $\lambda_{\underline{k}}=\lambda_{\underline{k}}(\mathrm{~S})$ can be mapped into a monotonically increasing energy function by some optical dispersion model, i.e.:

$$
\varepsilon_{\underline{k}}^{-2}=1-\lambda_{\underline{k}} \quad \text { (Drude's) }
$$

Energy will vary with respect to the barycenter values $\varepsilon_{\underline{k} s} \equiv$ $\varepsilon\left(\lambda_{\underline{k} s}\right)$ of the set of spherical multipoles, i.e. $\varepsilon_{\underline{k} s} \rightarrow \varepsilon_{\underline{k}}+$. We can model the topology change by introducing some excess charge at the face centers of the cube, with $S=1$ taken on as a reference state. This makes our problem equivalent to the crystal field splitting of 1-electron orbitals in the cubic-symmetry potential of an octahedral disposition with point group $O_{h} .{ }^{42}$ The numerical analysis of the trends of eigenvalues in Figure (2) upon $\mathrm{S}=0 \rightarrow 0^{+}$confirms such a spectroscopic analogy. Once eigenvalues are mapped into the energy equation (5), the diagrams in Figure (4) are obtained, giving a pictorial representation of the electromagnetic analog of crystal field splitting. In a nutshell, when shape changes, geometric eigenvalues change correspondingly as atomic energy levels in Tanabe-Sugano correlation diagrams.

It may be useful to shortly remind the difference between plasmon hybridization and crystal field plasmon splitting. Both concepts stem originally from quantum chemistry and manifest themselves in the form of energy level diagrams. The former points out a mixing of different energy levels, where primitive plasmons (symmetrically or anti-symmetrically) couple to give rise to hybridized plasmons of the joint system, and the final diagram resembles the energy's for molecular orbital formation. Crystal field theory instead is concerned with how degeneracy is split for a given orbital level with symmetry group assigned. For each (plasmon energy) eigenvalue, degeneracy is splitting and evolving as a function of particle topology.

In the present case, for $\mathrm{S}=0 \rightarrow 0^{+}$, we first note that the $x, y, z$ symmetries of $P$ states $(\ell=1)$ disallow a degeneracy removal:

$$
\varepsilon_{+}\left\{x_{k}\right\}=\varepsilon_{+}\left\{x_{h}\right\} \quad(P)
$$

while enquiring the cases $\ell=2,3,4$ allows to identify the follow- 
ing phenomenological series:

$$
\begin{aligned}
& \varepsilon_{+}\left\{x_{k} x_{h}\right\}<\varepsilon_{+}\left\{x^{2}-y^{2}, z^{2}\right\} \\
& \varepsilon_{+}\{x y z\}<\varepsilon_{+}\left\{x_{k}\left(x_{h}^{2}-x_{q}^{2}\right)\right\}<\varepsilon_{+}\left\{x_{k}\left(x_{k}^{2}-r^{2}\right)\right\} \\
& \varepsilon_{+}\left\{\left(z^{2}-r^{2}\right)\left(x^{2}-y^{2}\right),\left(x^{2}-r^{2}\right)\left(y^{2}-z^{2}\right)-\left(y^{2}-r^{2}\right)\left(z^{2}-x^{2}\right)\right\} \\
& <\varepsilon_{+}\left\{x_{k} x_{h}\left(x_{k}^{2}-x_{h}^{2}\right)\right\}<\varepsilon_{+}\left\{x^{4}+y^{4}+z^{4}-r^{4}\right\}
\end{aligned}
$$

subindices $\underline{k}$ and coefficients of orbital polynomials ${ }^{43}$ being omitted for simplicity, with $x_{k}, x_{h}, x_{q} \in\{x, y, z\}$.

All of these relationships agree with the predictions for octahedral field splitting, ${ }^{44,45}$ i.e. in Mullikan's notation:

$$
\begin{aligned}
& \varepsilon\left(T_{2 g}\right)<\varepsilon\left(E_{g}\right) \\
& \varepsilon\left(A_{2 g}\right)<\varepsilon\left(T_{2 g}\right)<\varepsilon\left(T_{1 g}\right) \\
& \varepsilon\left(T_{2 g}\right)<\varepsilon\left(E_{g}\right)<\varepsilon\left(T_{1 g}\right)<\varepsilon\left(A_{1 g}\right)
\end{aligned}
$$

where, in parentheses, are the states representative of irreducible representations of $O_{h}$, with degeneracy values that exactly correspond to equations (7), i.e. $\operatorname{deg}\left(A_{g}\right)=1, \operatorname{deg}\left(E_{g}\right)=2$ and $\operatorname{deg}\left(T_{g}\right)=3 .{ }^{44}$ The eleven states of $H$ series split into 2,3,3,3 levels (from lower to higher energies), which agree with the symmetry group properties of atomic orbitals with $\ell=5 .^{43}$

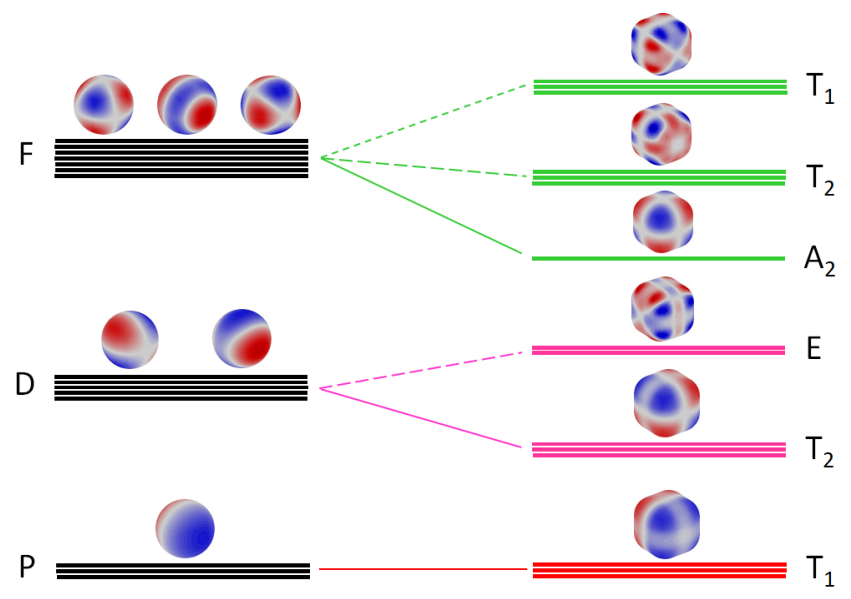

Fig. 4 Scheme of the plasmon splitting mechanism, in a potential of octahedral symmetry $\left(O_{h}\right)$, implied by CFT (image not in energy scale). State degeneracies are $\operatorname{deg}\left(A_{g}\right)=1, \operatorname{deg}\left(E_{g}\right)=2$ and $\operatorname{deg}\left(T_{g}\right)=3(\mathrm{~g}=$ gerade). It is important to realize that colors were chosen as in Figure (2). While plasmon hybridization mixes different energy levels, crystal field theory predicts if and how degeneracy is split for any orbital with a given symmetry.

At larger $S$ values, $\lambda_{\underline{k}}$ are not monotonic thereby reflecting a more general (and complex) symmetry behavior that requires a more precise quantitative description. This is not surprising, as the tetrahedral $T_{d}$ is another relevant cubic group in our context. While degeneracy of $P$ states won't still be removed upon $T_{d}$, an extended law should explain the increasing trend of $\lambda_{k}$ with increasing $\mathrm{S}(\ell>1)$. As it is usual in CFT, equation (4) may be generalized by projecting the potential onto the implied bra-ket eigenmodes. $V_{i}$ is ascribed to the spherically symmetric monopole produced at a radius $L / 2$ by an innermost pointwise charge $q_{i}$, e.g. in Gaussian units and with the conventions specified at the end of the former paragraph:

$$
\left\langle 00\left|V_{i} \partial_{\mathbf{n}} V_{i}\right| 00\right\rangle \equiv \frac{1}{2} \int Y_{00}^{*} Y_{00} \partial_{\mathbf{n}}\left(V_{i}^{*} V_{i}\right) d s=2 \frac{q_{i}^{2}}{L}
$$

where the complex conjugate of $V_{i}$ guarantees a real-valued functional. To get the numerator in equation (4), we remind the most general form the real part of octahedral, tetrahedral and cubic potentials assume, ${ }^{42}$ and write (SI section 6I):

$$
V_{\ell}(\mathbf{r})=\frac{q_{e}}{r} \sum_{\ell^{\prime} \in 2 \mathrm{~N} \backslash 2}^{2 \ell} \frac{\mathrm{L}^{\ell^{\prime}}}{\mathrm{L}_{\ell \ell^{\prime}}} \quad(\ell \geq 1)
$$

Here, $q_{e}$ is the effective charge generating $V_{e}$, the spatial average in the sum is performed over a radial wave function $\left(\overline{r^{0}}=1\right)$ and $\mathrm{L}_{\ell \ell^{\prime}}$ is a linear superposition of the form $\mathrm{L}_{\ell \ell^{\prime}}=\mathrm{L}_{\ell \ell^{\prime}}\left\{Y_{\ell^{\prime} 0}, Y_{\ell^{\prime} \pm 4}\right\}$. We then get eigenvalues from equation (4) by regarding $V_{e}=V_{\ell}$ into:

$$
\lambda_{\underline{k} b}=\frac{\left\langle X_{\underline{k}}\left|V_{e} \partial_{\mathbf{n}} V_{e}\right| X_{\underline{k}}\right\rangle}{\left\langle 00\left|V_{i} \partial_{\mathbf{n}} V_{i}\right| 00\right\rangle}
$$

that is clearly truncated to $\ell^{\prime} \leq \ell$ and, from symmetry arguments, doesn't display any term for $\ell^{\prime}=2\left(\mathrm{~L}_{\ell 2} \equiv 0\right) .{ }^{44}$ Cubic harmonics $X_{\underline{k}}$, i.e. linear combinations of real $Y_{\ell m}$ at fixed $\ell$, form the natural basis in irreducible representations of cubic groups.

To highlight the role of shape descriptor when an arbitrary surface is concerned, we resort to the mean value theorem for definite integrals (SI section 6L). The particle is divided into cubic $(c)$, spherical $(s)$ and semicubic $(b)$ domains, each contributing (in units of $6 L^{2}$ ) to the overall area as $a_{c}=S^{2}, a_{s}=\frac{\pi}{6}(1-S)^{2}$, $a_{b}=\frac{\pi}{2} S(1-S)$. Let $f$ be a continuous function onto a compact and rectifiable surface, the following notation is employed:

$$
\frac{1}{6 L^{2}} \int f d s=\langle f\rangle_{c} a_{c}+\langle f\rangle_{s} a_{s}+\langle f\rangle_{b} a_{b}
$$

the effective charge $\left(q_{e}\right)$ variation from sphere to cube obeying a minimal, linear and homogeneous, description:

$$
q_{\underline{k} b}(\mathrm{~S})=q_{\underline{k} s}+\left(q_{\underline{k} c}-q_{\underline{k} s}\right) \mathrm{S}
$$

In comparison to the purely spherical symmetry, with $q_{i}=q_{e}(\mathrm{~S}=$ 0 ), a change of shape in CFT is carrying a charge displacement. In light of equations (12) and (13), the eigenvalue equation (11) can be written as a polynomial in $\mathrm{S}$ :

$$
\lambda_{\underline{k} b}=-\left(z_{\underline{k} s}+z_{\underline{k} b} \mathrm{~S}\right)^{2}\left[\sigma_{\ell m}(1-\mathrm{S})^{2}+\mu_{\ell m} \mathrm{~S}(1-\mathrm{S})+\chi_{\ell m} \mathrm{~S}^{2}\right]
$$

where, for any mode, $z_{\underline{k} s}$ and $z_{\underline{k} b}$ express $q_{\underline{k} s}$ and $q_{\underline{k} c}-q_{\underline{k} s}$ in unit of charge $q_{i}$. Coefficients $\sigma_{\ell m}, \mu_{\ell m}, \chi_{\ell m}>0$ are, respectively, averages of the spherical, semicubic and cubic contributions to $\lambda_{\underline{k} b}$, whose explicit expressions can be found in SI section (6M). Because of the tough calculations involved (Clebsh-Gordan coefficients and integration over arbitrary surfaces), their numerical determination falls beyond the aims of this study, but equation (14) specifies a minimal fourth-degree polynomial well fitting the eigenvalues in the whole S domain with $\chi_{\ell m}, \mu_{\ell m}, \sigma_{\ell m} \in(0.4-1.3), z_{\underline{k} s} \in(1-2)$, $z_{k b} \in(0-1)$ (see SI section $\left.6 \mathrm{~N}\right)$. We observed that the charge de- 
fect $z_{\underline{k} b} / z_{\underline{k} s}=q_{\underline{k}} / q_{\underline{k} s}-1$ tends to be negligible in dipole $\left(\leq 5 \cdot 10^{-3}\right)$ and quadrupole modes $\left(\leq 5 \cdot 10^{-2}\right)$, suggesting the topology contribution of the dominant modes to be only slightly dependent on geometry, and justifying further the factorization in equation (14).

Non-retarded and retarded analog of crystal field splitting. In a quasistatic picture, equation (14) affords a topological interpretation of LSP resonance, as Drude's model predicts $\lambda \propto \sqrt{1-\lambda}$ (equation 5). When surface effects are discarded, we expect from the only topological charge term:

$$
\frac{\sqrt{\Delta \hbar^{2}}}{\lambda_{0}} \approx \bar{z}_{s}+\bar{z}_{b} \mathrm{~S} \quad(\text { non }- \text { retarded })
$$

where, at some wavelength scale $t_{0}$, one has $\Delta t^{2} \equiv \lambda^{2}-\lambda_{0}^{2}$, with $\lambda$ being the resonance wavelength. Note that a behavior more or less deviating from linearity depends on the entity of $\lambda / \lambda_{0}$. Equation (15) has been applied to quasistatic LSP values (dataset in column qs of Table S2 in SI section 5G) under two different conditions. In a first, the best-fit was constrained to the $\bar{z}_{k}$ ranges extrapolated numerically from the analysis of multipole coefficients at different $(\ell, m)$ (see former paragraph), returning an almost linear behavior. In the second, unconstrained, the LSP resonant wavelength gains a little more precision at the expense of a mild non-linearity. The two interpolations are shown in Figure (5), both in satisfactory agreement with the computed dataset.

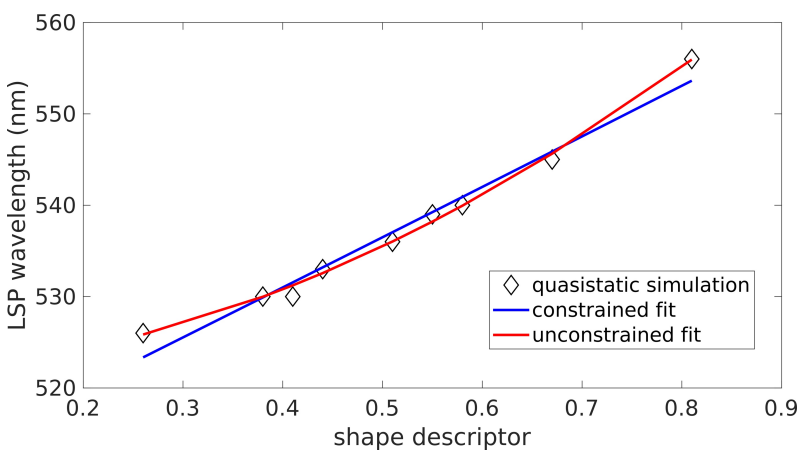

Fig. 5 Quasistatic LSP wavelength $\lambda$ (qs) vs shape descriptor S (points). Blue and red lines (equation 15) respectively for $\bar{z}_{s}=1.65, \bar{z}_{b}=0.24, \lambda_{0}=$ $236.7 \mathrm{~nm}\left(R^{2}=0.97\right)$, and $\bar{z}_{s}=9.9 \cdot 10^{-3}, \bar{z}_{b}=0.44, \lambda_{0}=521.8 \mathrm{~nm}\left(R^{2}=\right.$ $0.99)$.

To include retardation in CFT by analytical means may be rather demanding, especially for non-spherical shapes. However, with some judicious choice of retarded fields, an evaluation of finite-size effects may be provided, even though remarkable corrections are not expected. Spherical $\ell$-modes should get (meaningfully) retarded when $\ell<L /(2 \lambda)$, ${ }^{46}$ which here is far from being satisfied. On adopting Coulomb's gauge, SI section (6O) reports a thorough analysis of retardation effects in light of CFT, and here the two main results follow. For the spherical symmetry, calculations can be specialized at $R=L / 2$ and the first corrected eigenvalue reads:

$$
\lambda_{\ell=1}^{\text {ret }}=-2\left[1+(k R)^{2}+\mathrm{i}(k R)^{3}\right]+\mathrm{O}(k r)^{4} \quad(\text { sphere })
$$

in fair agreement with previous corrections from radiation damp- ing and depolarization, ${ }^{47}$ implying $\delta \lambda_{\ell=1}^{\text {ret }}=-3(k R)^{2}-2 \mathrm{i}(k R)^{3}$, and with the second-order term ${ }^{48}$ coming from an analysis of Mie's scattering coefficients, $-\frac{12}{5}(k R)^{2}$.

Concerning semicubic eigenvalues, it is proven in SI section (6O) that the numerator in equation (11) transforms as $\partial_{\mathbf{n}} V_{e} \rightarrow-\mathrm{i} k \mathbf{n}$. $\mathbf{A}^{\text {ret }}$, where $\mathbf{A}^{\text {ret }}$ is the retarded vector potential. Accordingly, the retardation correction reads:

$$
\Delta \lambda_{\mathrm{ret}}^{2}=\left[\frac{1}{66} \frac{\overline{r^{4}}}{R^{* 4}}\left(23+\frac{2527}{39} \frac{\overline{r^{4}}}{R^{* 4}}\right) \Delta \lambda^{2}+2 \lambda_{0}^{2}\right]\left(k R^{*}\right)^{2}+\mathrm{O}\left(k R^{*}\right)^{3}
$$

with $\Delta t_{\text {ret }}^{2}=\lambda^{\text {ret }}{ }^{2}-\lambda^{2}$ being the second-order difference between the squares of retarded and non-retarded wavelengths, while $R^{*}=R^{*}(\mathrm{~S})$ denotes the radius of a spherical volume equivalent to the semicube's. To use the last relation simply requires to recover the non-retarded best fit (equation 15) and the expression $R^{*}(\mathrm{~S})=(29.92 \mathrm{~S}+11.80) \mathrm{nm}$ (SI section 5F). In Figure (6) are two applications, one to the retarded-geometrically corrected data (sc), the second to the experimental measurements (ex). We remind that the latter are affected by an excess charge injection term $(\xi)$, increasing the plasma frequency by a factor of $\sqrt{1+\xi}$. The wavelength $\lambda_{0}$ thus was lowered accordingly, based on the expression $\xi(\mathrm{S})=0.213 \mathrm{~S}-0.041$ best fitting the numerical computations (SI section 5F). The average spatial scale, which in the original CFT roughly identifies Bohr's radius, ${ }^{42} \overline{r^{4}} \sim a_{0}^{4}$, here is expected to range in the nm scale (some lattice constants). Best fits return in fact $\overline{r^{4}} \sim 1 \mathrm{~nm}^{4}$ in both circumstances (see caption to Figure 6).

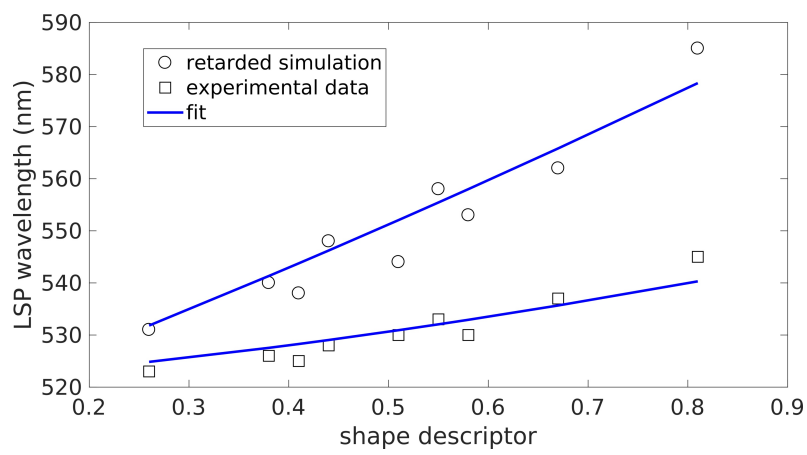

Fig. 6 Retarded LSP wavelength $\lambda^{\text {ret }}$ vs shape descriptor S (points), based on the interpolation (blue line) in Figure (5) and corrected by equation (17). Upper line interprets the retarded and geometrically corrected data, $\lambda(\mathrm{sc})$ in Table (S2) (SI section 5G), with $k=11.4 \mu \mathrm{m}^{-1}$. Lower line fits the experimental data, $\lambda$ (ex), where $k=11.8 \mu \mathrm{m}^{-1}$ and $\lambda_{0}$ was excess-charge corrected (see text). Best fits yield $\overline{r^{4}}=0.98\left(R^{2}=0.91\right)$ and $0.99 \mathrm{~nm}^{4}\left(R^{2}=0.86\right)$, respectively.

Eigenvalues and hybridization in cubes. The accuracy best fits were conducted with (average determination coefficient $=0.99$ ) is encouraging to get reliable extrapolations of cube eigenvalues $\left(\lambda_{\underline{k} b} \rightarrow \lambda_{\underline{k} c}\right.$ for $\left.\mathrm{S} \rightarrow 1^{-}\right)$, as they can suffer from numerical instabilities due to wedge- and vertex-like effects.

Values of $\lambda_{\underline{k} c}$ are inferred from polynomial coefficients in SI section $(6 \mathrm{~N})$ and classified in Tables (S3,S4) in terms of the spherical $\ell$, the degeneracy of $m$ states and their symmetry character. These trends agree with former studies on resonating cubes, ${ }^{36,39,49}$ and seem to suggest hybridization of a number of $\ell$-bands (e.g. see 
$\ell=5)$.

Table 2 Cube eigenvalues extrapolated from the first five spherical $\ell$ values.

\begin{tabular}{|llll|llll|}
\hline$\ell$ & deg & sym & $-\lambda_{\underline{k} c}$ & $\ell$ & deg & sym & $-\lambda_{\underline{k}}$ \\
\hline 1 & 3 & $\mathrm{z}$ & 4.00 & 3 & 3 & $\mathrm{xy}$ & 2.23 \\
2 & 3 & $\mathrm{xy}$ & 3.97 & 4 & 3 & $\mathrm{xyz}, \mathrm{x}^{2}-y^{2}$ & 2.17 \\
3 & 1 & $\mathrm{xyz}$ & 3.96 & 5 & 3 & $\mathrm{xy}, \mathrm{x}^{2}-y^{2}$ & 2.16 \\
4 & 1 & $\mathrm{xyz}$ & 3.37 & 5 & 2 & $\mathrm{z}$ & 2.12 \\
3 & 3 & $\mathrm{z}$ & 2.79 & 4 & 2 & $\mathrm{xy}$ & 1.96 \\
4 & 3 & $\mathrm{xy}$ & 2.74 & 5 & 1 & $\mathrm{xy}$ & 1.90 \\
2 & 2 & $\mathrm{x}^{2}-y^{2}$ & 2.36 & 5 & 2 & $\mathrm{z}, \mathrm{x}^{2}-y^{2}$ & 1.88 \\
5 & 3 & $\mathrm{z}, \mathrm{x}^{2}-y^{2}$ & 2.36 & & & & \\
\hline
\end{tabular}

Conclusions. An analysis of spontaneous electric oscillations is carried out in light of crystal (or ligand) field theory (CFT), allowing a reinterpretation of geometric eigenmodes and the resonant plasmon wavelength in terms of a simple shape descriptor (S). This relationship is promising to better understand the role of shape in plasmonics and nanophotonics, continuing a cross-fertilization with theoretical chemistry started almost two decades ago with particle hybridization. ${ }^{50,51}$ An essential trait of CFT is that a shape or geometric perturbation alters the energy levels giving rise to Tanabe-Sugano correlation diagram. If the starting configuration is spherical, energies at a fixed $l$ will no longer be isoenergetic. Accordingly, a plasmon splitting concept is inferred from the electromagnetic analog of CFT, both in a nonretarded (i.e. electrostatic) and retarded picture. From a broader prospect, this research is still in its infancy. The insights here provided, for instance, have important implications in assessing the exact nanocrystal topology by merely optical means. In addition, the well-resolved relation between maxima of LSP resonance and shape descriptor can serve as a useful tool in plasmon-based biosensing, as it either relies on refractive index changes ${ }^{52,53}$ or nanocrystal shape transitions in response to a certain degree of oxidative stress in a given medium. ${ }^{54}$

\section{Associated Content}

\section{Supporting Information}

The Supporting Information (SI) is available free of charge on the ACS Publications website at DOI:

- SI section 1: Methods (Experimental; Numerical).

- SI section 2: Statistical Analysis of Geometric Nanoparticle Features - A. Shape Descriptor from Circularity Values; B. Statistical Correction to Circularity; C. Statistics of Radii.

- SI section 3: Geometric Eigenmodes.

- SI section 4: Multipole contributions to LSP spectra in Ag particles.

- SI section 5. Inquiring Anomalous Redshift Sources - D. Dielectric Constant Reduction upon Ion Concentration; E. Geometric Polydispersity; F. Free and Bound Surface Charges; G. Summary of all LSP Resonance Data

- SI section 6: Mathematical Details - H. Sphere Eigenvalues from Vanishing Electrostatic Energy; I. Basic References to
Crystal (Ligand) Field Theory (CFT); L. Mean Value Theorem for Definite Surface Integral; M. CFT Coefficients in the Geometric Eigenvalue Equation; N. Best Fitting Eigenvalues vs Shape Descriptor; O. Retardation Calculations.

\section{Author Information}

\author{
Corresponding Author \\ Email: marek.grzelczak@dipc.org \\ Email: Jordi.Sancho.Parramon@irb.hr \\ ORCID
}

Stefano A. Mezzasalma: 0000-0002-2968-7497

Marek Grzelczak: 0000-0002-3458-8450

Jordi Sancho-Parramon: 0000-0003-3284-8666

\section{Notes}

The authors declare no competing financial interest.

\section{Funding Sources}

This work was supported by the Ministry of Environment and Energy, the Ministry of Science and Education, the Environmental Protection and Energy Efficiency Fund, and the Croatian Science Foundation (PKP-2016-06-4469). S.A.M. thanks DIPC (San Sebastian, Spain), IRB (Zagreb, Croatia) and LINXS (Lund, Sweden) for kind hospitality and financial support.

\section{Acknowledgements}

Ana Sánchez Iglesias is kindly acknowledged for her help during the synthesis of nanoparticles.

\section{Author Contributions}

The authors equally contribute to this work (M.G. by the experimental part, J.S.P. by the computational session, S.A.M. by the theoretical formulation).

\section{References}

1 Grzelczak, M.; Pérez-Juste, J.; Mulvaney, P.; Liz-Marzán, L. M. Shape control in gold nanoparticle synthesis. Chem. Soc. Rev. 2008, 37, 1783-1791, 01449.

2 Myroshnychenko, V.; Rodríguez-Fernández, J.; PastorizaSantos, I.; Funston, A. M.; Novo, C.; Mulvaney, P.; LizMarzán, L. M.; García de Abajo, F. J. Modelling the optical response of gold nanoparticles. Chem. Soc. Rev. 2008, 37, 17921805.

3 Novo, C.; Funston, A. M.; Mulvaney, P. Direct observation of chemical reactions on single gold nanocrystals using surface plasmon spectroscopy. Nat. Nanotechnol. 2008, 3, 598-602.

4 Kelly, K. L.; Coronado, E.; Zhao, L. L.; Schatz, G. C. The Optical Properties of Metal Nanoparticles: The Influence of Size, Shape, and Dielectric Environment. J. Phys. Chem. B 2003, 107, 668-677.

5 Brongersma, M. L.; Halas, N. J.; Nordlander, P. Plasmoninduced hot carrier science and technology. Nat. Nanotechnol. 2015, 10, 25-34.

6 Brown, A. M.; Sundararaman, R.; Narang, P.; Goddard, W. A.; Atwater, H. A. Nonradiative Plasmon Decay and Hot Carrier 
Dynamics: Effects of Phonons, Surfaces, and Geometry. ACS Nano 2016, 10, 957-966.

7 Ma, X.-C.; Dai, Y.; Yu, L.; Huang, B.-B. Energy transfer in plasmonic photocatalytic composites. Light Sci. Appl. 2016, 5, e16017.

8 Bansil, A.; Lin, H.; Das, T. Colloquium: Topological band theory. Rev. Mod. Phys. 2016, 88, 021004.

9 Pfeiffer, C. A.; Economou, E. N.; Ngai, K. L. Surface polaritons in a circularly cylindrical interface: Surface plasmons. Phys. Rev. B 1974, 10, 3038-3051.

10 Stockman, M. I. Nanofocusing of Optical Energy in Tapered Plasmonic Waveguides. Phys. Rev. Lett. 2004, 93, 137404.

11 Veronis, G.; Fan, S. Bends and splitters in metal-dielectricmetal subwavelength plasmonic waveguides. Appl. Phys. Lett. 2005, 87, 131102.

12 Shitrit, N.; Bretner, I.; Gorodetski, Y.; Kleiner, V.; Hasman, E. Optical Spin Hall Effects in Plasmonic Chains. Nano Lett. 2011, 11, 2038-2042.

13 Jensen, J.; Sigmund, O. Topology optimization for nanophotonics. Laser Photonics Rev. 2011, 5, 308-321.

14 West, P.; Ishii, S.; Naik, G.; Emani, N.; Shalaev, V.; Boltasseva, A. Searching for better plasmonic materials. Laser \& Photonics Reviews 2010, 4, 795-808.

15 Bassani, F.; Pastori Parravicini, G. Electronic States and Optical Transitions in Solids; Pergamon, New York, 1975.

16 Mezzasalma, S. A.; Janicki, V.; Salamon, K.; SanchoParramon, J. Combination Law for Drude-Sommerfeld's Electron Damping in Multilayer Thin Metal Films. Phys. Status Solidi RRL 2018, 12, 1800149.

17 DeWitt, B. S. Dynamical Theory in Curved Spaces. I. A Review of the Classical and Quantum Action Principles. Rev. Mod. Phys. 1957, 29, 377-397.

18 da Costa, R. C. T. Quantum mechanics of a constrained particle. Phys. Rev. A 1981, 23, 1982-1987.

19 Ikegami, M.; Nagaoka, Y.; Takagi, S.; Tanzawa, T. Quantum Mechanics of a Particle on a Curved SurfaceComparison of Three Different Approaches. Progr. Theor. Phys. 1992, 88, 229-249.

20 Cantele, G.; Ninno, D.; Iadonisi, G. Topological surface states in deformed quantum wires. Phys. Rev. B 2000, 61, 1373013736.

21 Marchi, A.; Reggiani, S.; Rudan, M.; Bertoni, A. Coherent electron transport in bent cylindrical surfaces. Phys. Rev. B 2005, 72, 035403.

22 Batz, S.; Peschel, U. Linear and nonlinear optics in curved space. Phys. Rev. A 2008, 78, 043821.

23 Valle, G. D.; Longhi, S. Geometric potential for plasmon polaritons on curved surfaces. J. Phys. B 2010, 43, 051002.

24 Cui, X.; Lai, Y.; Qin, F.; Shao, L.; Wang, J.; Lin, H.-Q. Strengthening Fano resonance on gold nanoplates with gold nanospheres. Nanoscale 2020, 12, 1975-1984.

25 García de Abajo, F. J.; Aizpurua, J. Numerical simulation of electron energy loss near inhomogeneous dielectrics. Phys. Rev. B 1997, 56, 15873-15884.
26 García de Abajo, F. J.; Howie, A. Retarded field calculation of electron energy loss in inhomogeneous dielectrics. Phys. Rev. B 2002, 65, 115418.

27 Rodríguez-Fernández, J.; Pérez-Juste, J.; Mulvaney, P.; LizMarzán, L. M. Spatially-Directed Oxidation of Gold Nanoparticles by Au(III)CTAB Complexes. J. Phys. Chem. B 2005, 109, 14257-14261.

28 Mayergoyz, I. D.; Fredkin, D. R.; Zhang, Z. Electrostatic (plasmon) resonances in nanoparticles. Phys. Rev. B 2005, 72, 155412.

29 Hohenester, U.; Trügler, A. MNPBEM-A Matlab toolbox for the simulation of plasmonic nanoparticles. Comp. Phys. Comm. 2012, 183, 370-381.

30 De Abajo, F. G.; Howie, A. Relativistic electron energy loss and electron-induced photon emission in inhomogeneous dielectrics. Phys. Rev. Lett. 1998, 80, 5180.

31 Nicoletti, O.; de La Peña, F.; Leary, R. K.; Holland, D. J.; Ducati, C.; Midgley, P. A. Three-dimensional imaging of localized surface plasmon resonances of metal nanoparticles. Nature 2013, 502, 80.

32 Mezzasalma, S.; Baldovino, D. Characterization of Silicon Nitride Surface in Water and Acid Environment: A General Approach to the Colloidal Suspensions. J. Colloid Interface Sci. 1996, 180, 413 - 420 .

33 Bohren, C. F.; Hunt, A. J. Scattering of electromagnetic waves by a charged sphere. Can. J. Phys. 1977, 55, 1930-1935.

34 Olmon, R. L.; Slovick, B.; Johnson, T. W.; Shelton, D.; Oh, S.H.; Boreman, G. D.; Raschke, M. B. Optical dielectric function of gold. Phys. Rev. B 2012, 86, 235147.

35 Johnson, P. B.; Christy, R.-W. Optical constants of the noble metals. Phys. Rev. B 1972, 6, 4370.

36 Langbein, D. Normal modes at small cubes and rectangular particles. J. Phys. A: Math. Gen. 1976, 9, 627.

37 van Gelder, A. P.; Holvast, J.; Stoelinga, J. H. M.; Wyder, P. Localized polariton modes of small cubic crystals. J. Phys. C 1972, 5, 2757.

38 Ouyang, F.; Isaacson, M. Surface plasmon excitation of objects with arbitrary shape and dielectric constant. Philos. Mag. B 1989, 60, 481-492.

39 Hohenester, U.; Krenn, J. Surface plasmon resonances of single and coupled metallic nanoparticles: A boundary integral method approach. Phys. Rev. B 2005, 72, 195429.

40 B, W. A. Symmetry and Quantum Systems; Van Nostrand Reinhold, New York, 1977.

41 Bethe, H. A. Splitting of terms in crystals. Ann. Physik 1929, 3, 133-206.

42 Figgis, B. N.; Hitchman, M. A. Ligand Field Theory and its Applications; New York: Wiley-VCH, 2000.

43 B. King, R. Chemical Applications of Topology and Group Theory. 31. Atomic Orbital Graphs and the Shapes of the $g$ and $h$ Orbitals 1. J. Phys. Chem. A 1997, 101, 4653-4656.

44 Schläfer, H. L.; Gliemann, G. Basic Principles of Ligand Field Theory; Wiley-Interscience, New York, 1969.

45 Perumareddi, J. R. Splitting of Terms Beyond a D-Term in an 
Octahedral Ligand Field. Z. Naturforsch. 1981, 36a, 735-742.

46 Fuchs, R.; Kliewer, K. L. Optical Modes of Vibration in an Ionic Crystal Sphere. J. Opt. Soc. Am. 1968, 58, 319-330.

47 Zeman, E.; Schatz, G. An accurate electromagnetic theory study of surface enhancement factors for $\mathrm{Ag}, \mathrm{Au}, \mathrm{Cu}, \mathrm{Li}, \mathrm{Na}$, Al, Ga, In, Zn, and Cd. J. Phys. Chem. 1987, 91, 634-643.

48 Bohren, C.; Huffman, D. R. In Absorption and Scattering of Light by Small Particles; Bohren, C., Huffman, D. R., Eds.; New York: Wiley-VCH, 1998.

49 Fuchs, R. Theory of the optical properties of ionic crystal cubes. Phys. Rev. B 1975, 11, 1732-1740.

50 Prodan, E.; Radloff, C.; Halas, N. J.; Nordlander, P. A Hybridization Model for the Plasmon Response of Complex Nanostructures. Science 2003, 302, 419-422.

51 Sancho-Parramon, J.; Bosch, S. Dark modes and Fano resonances in plasmonic clusters excited by cylindrical vector beams. ACS Nano 2012, 6, 8415-8423.

52 Lee, Y. H.; Chen, H.; Xu, Q.-H.; Wang, J. Refractive Index Sensitivities of Noble Metal Nanocrystals: The Effects of Multipolar Plasmon Resonances and the Metal Type. J. Phys. Chem. C 2011, 115, 7997-8004.

53 Chen, H.; Kou, X.; Yang, Z.; Ni, W.; Wang, J. Shape- and SizeDependent Refractive Index Sensitivity of Gold Nanoparticles. Langmuir 2008, 24, 5233-5237.

54 Coronado-Puchau, M.; Saa, L.; Grzelczak, M.; Pavlov, V.; LizMarzán, L. M. Enzymatic modulation of gold nanorod growth and application to nerve gas detection. Nano Today 2013, 8, 461-468, 00033. 